\title{
MECHATRONICS, ROBOTICS AND COMPONENTS FOR AUTOMATION AND CONTROL IFAC CC MILESTONE REPORT
}

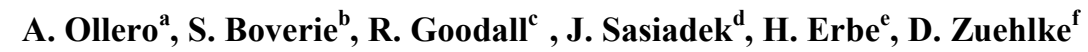 \\ ${ }^{a}$ Dept. Ingeniería de Sistemas y Automática, E.S.Ingenieros, Univ Sevilla \\ Camino de los Decubrimientos. Spain \\ ${ }^{b}$ Siemens Automotive, BP 1149, 1, Ave.du Mirail. F-31036 Toulouse CEDEX. France \\ ${ }^{c}$ El. Engineering. Dept. Loughborough University, Loughborough LE11 3TU. UK \\ ${ }^{d}$ Dept. of Mechanical \& Aerospace Eng. Carleton Univ. 1125 Colonel by Dr. Ottawa \\ ONT K1S 5B6. Canada \\ ${ }^{e}$ Technische Univ. Berlin. Inst. F. Berufliche Bildung, Zentrum Mensch-Maschine-Systeme \\ Sekr. FR 4-4. Franklinstrasse 28/29. D-10587 Berlin. Germany \\ ${ }^{f}$ Institute of Production Automation, University of Kaiserslautern, \\ P.O. Box 3049,67653 Kaiserslautern, Germany
}

\begin{abstract}
This paper is devoted to the analysis of a broad technological field of Mechatronics, Robotics and Components for automation and control systems. Several subfields are considered: i) Components and instruments, involving sensors, actuators, embedded systems and communications; ii) Mechatronics concepts and technologies; iii) Robotics; iv) Human-machine systems, including technical issues and social implications; and v) Cost-oriented automation which is a multidisciplinary field involving theory, technologies and application as well as economical and social issues. First current key problems in this field are considered then, the accomplishment and trends are analysed. Finally, the forecast is presented. Copyright (C) 2005 IFAC
\end{abstract}

Keywords: Components for control, Mechatronic Systems, Robotics, Cost Oriented Automation, Human-Machine Systems.

\section{INTRODUCTION}

Control technologies and applications have experienced highly significant developments in the last 10 years. This development has been driven by progress in innovative sensors and actuators, and the increasing performance of computer systems, including embedded systems for control.

In addition, recent developments of communication technologies have also led to novel distributed control technologies, including systems with wire and wireless communications in the control loops and networked systems with multiple interconnected objects. The application domains are very large; including process control with very complex interconnected systems, factory automation as well as robotics, building automation, and transportation systems, which is today one of the most "pushing" domains.

This paper pays significant attention to the field of Components and Instruments for control including sensors, actuators, embedded systems and communications.

This paper also devotes particular attention to Mechatronic Systems. For many years control engineers have been using sensors and electronic processing to enhance and/or alter the performance 
of mechanical systems, in many cases to provide a level of functionality that is not possible without the electronics. The sophistication which has more recently been possible through increasingly powerful processing devices and heightened software skills has resulted in an increasing trend towards embedded mechatronic solutions involving a synergetic combination of mechanics, electronics, software and computing. This necessitates a multi-disciplinary understanding of the relevant scientific and engineering principles, and the individual knowledge of the mechatronic engineer must be sufficiently comprehensive to be able to create the innovative combination that makes up mechatronic solutions.

Robotics is a relatively new field in science and engineering. The broadly accepted definition is that robotics is an intelligent connection of perception to action. Robot perception is performed by sensors. Sensors allow robots to sense and interact with the changing environment. Information acquired by the robot is then processed using intelligent algorithms. The resulting commands are passed on to the actuators -devices that drive joints and actuate parts of the robots. This paper also focuses on Robotics.

The study of interactions between human and machines is an important aspect in the adoption of control technologies involving both technical issues and social implications. Human-machine studies consider all conditions where humans (individuals as well as groups) use, control or supervise tools, machines or technological systems. It fosters analysis, design, modeling and evaluation of Human Machine Systems (HMS) which includes: decision making and cognitive processes, modeling of human performance (reliability, mental work load, predictability), real and virtual environments, design methodology, task allocation-sharing and job design, intelligent interfaces, human operator support, work organization, and selection and training criteria. Over the last few decades a shift from the more hardware oriented HMS-topics to the more software and system oriented topics is recognized, which may be characterized by cognitive ergonomics. All these aspects related to human-machine interactions are also considered in the paper.

Finally, it should be noted that the paper also discusses the implications of Cost Oriented Automation, or affordable automation, which is a cross-sectoral field involving theory, technology, and application as well as economical and social issues. Therefore the focus is not upon cheap components and instruments for control tasks, but upon the lifecycle of automation systems regarding costeffectiveness. Maintenance is a crucial point, and therefore has to be considered integrated with a performance-oriented product- and processdevelopment. An agile human-human and humanmachine collaboration to fulfil this demands has to be developed.
This paper is an update of the previously published report (Ollero et al, 2002) originally presented at the15th IFAC World Congress. It presents new developments and trends in the above fields. The remainder of the paper is organized as follows. The next section is devoted to the current key problems. Section 3 is devoted to the accomplishments and trends. The forecast of the involved technologies is presented in section 4 . Sections 5 and 6 are devoted to the conclusions and references.

\section{CURRENT KEY PROBLEMS}

\subsection{Components and Instruments}

Partitioning a complex system into more manageable units is essential to cope with today's increasing complexity of process control and biomedical applications. In this set of units, intelligent sensors play an essential role in the operation of complex real-life distributed systems. The aim of intelligent sensors is to sample raw data in order to extract some information, where information can be defined as a measure of how well the data fit into information patterns. The field of large distributed control systems (like power plants and manufacturing processes) remains the main area of application of the intelligent components. The process monitoring and defect detection methods play a fundamental role to improve the quality of the result at reduced manufacturing costs.

As far as communications are concerned, Field Buses have been extensively used. These are special purpose networks that are basically used in real time applications for connecting field equipment and control devices. Field buses have been developed for more than 20 years by different companies or consortia. Today, they are one of the key components of automation systems, which is a good reason for the lack of current standardization, even though a lot of standards have been created (too many standards kill the concept of standard!). Current research topics are related to all the layers of the protocol stack, from the physical to the application one. The underlying problems in the study of wireless field buses are the connectivity of the stations, and the impact of perturbation on the upper protocol layers, and on the real time constraints. Ethernet systems can be also considered under the perspective of Field Buses. The main problems are: i) the determinism or the predictability of the communication and then of the application behaviour; and ii) the robustness of such distributed applications. In addition, the proof of properties (as stability, coherence or consistency) of networked controlled systems is one of the major research trends for the current decade. Despite a lot of local or specific solution elements are known, current modelling techniques are generally not suited to reach these goals, and the theory to prove distributed systems is rather weak. Thus a strong 
research effort should be dedicated in that direction within the next years

Components and instruments are likewise essential within the transportation/automotive application fields. For nearly a century the automobile has been the principal means of transport in the industrial world. The past twenty-five years have seen a profound evolution in behavior. This is linked, on one hand, to the awareness of automobiles contributing to the catastrophic rise in environmental pollution, and on the other hand to the continued search for driver comfort, safety, and security.

The implementation of severe regulations related to emission controls, fuel consumption, the search for improved drivability and output, as well as the improvement of safety in road traffic corresponds to an increasing demand from a majority of users as well as to a social and economical necessity. These new requirements have inevitably led to the need for more and more precise control of vehicle operation. Therefore, today, more and more vehicle functions are performed through strategies implemented in various type of electronic control units (ABS, ECU, Injection ECU, . . .). Over the last decade, the automobile became a very significant market for the electronics industry, accounting for more than 10 percent of the overall turnover. The electronic functions initially applied to the management of the engine have progressively encroached upon safety areas (ABS, air-bag, obstacle detection, etc.), security areas (access control, immobilizer), comfort areas (air conditioning), and of course driving aids.

The development of these new functions is related with the introduction in the automobile application field of more sophisticated sensor concepts (see Grace, 2002). These "intelligent" sensors will be able to provide synthetic information to the decision algorithms whether directly to the driver. It implies the adjunction to the "basic sensing device" of upper signal processing levels which allow to extract the pertinent information from a set of measurements, to fuse the information provided by several sensors, to classify them, ... (i.g; Vision sensor with dedicated image processing, radars with data extraction process etc, ...).

\subsection{Mechatronics}

The key problems for future developments in mechatronics focus around the multidisciplinary requirements of both engineers and the tools that they use. The actual creation of the system structure (i.e. the appropriate balance of form and function between the contributing disciplines) has been largely a human-dominated process that relies upon the insight and ingenuity of the engineer, and it is likely that this will continue to be the case. Nevertheless their endeavours need to be supported by usable, efficient, accurate design tools, and the availability of an effective design interface and easy access to multi-objective optimization techniques are critical. However there is a key issue related to the choice of models, and it's important to distinguish between the design model and the simulation model: the former is a simplified model used for synthesis of the control strategy and algorithm, whereas the latter is a full-complexity model to test the system performance. Modern model-based design approaches are often required to handle the complexity of the systems being developed, either for the controller itself or for estimators to access difficult or impossible to measure variables, in which case the controller and/or estimator assumes a dynamic complexity equal to or greater than that of the design model. The design model must therefore be sufficiently accurate for the purpose, but not so complex as to make implementation impossible.

Another issue arises because the integrity/reliability/availability required of the mechatronic solution is increasingly of general importance (i.e. not only for safety-related applications), and so a vital mechatronic skill is in the area of fault diagnosis and tolerance. Generally the integrity of mechanically-based products and processes can be assured by careful design of critical components, supported by targeted maintenance regimes, which as far as possible avoid dangerous failures. However failure modes for sensors, electronics and software are much more diverse and integrity now has to be provided through functional and analytical redundancy. The traditional approach for new solutions has often been: first create the outline scheme (i.e. the synergetic combination of mechanics, electronics, software and computing); then carry out detailed design of the controllers to meet performance requirements; then finally consider fault tolerance. However it is possible that the fault tolerance could have been achieved easier/better/cheaper had it been taken into account at the very first stage, and so a general theory and/or methodology for handling the synthesis problem for complex multi-disciplinary systems to meet multiple objectives and constraints, including those related to fault tolerance, is needed to maximize the impact of mechatronics.

A final key issue is related to human factors. Early mechatronic products required only limited human interaction, whereas their ever-broadening applicability means that many mechatronic products are now used on daily basis and human factors need to have correspondingly increased importance. In part this again comes back to the skill base of the mechatronic engineer, but also the contribution of the design tools needs careful consideration. This observation also links strongly with many of the ideas identified in Human-Machine Systems (see section 2.4). 


\subsection{Robotics}

The key problems in robotics are related to control systems, perception systems and advanced mechanical design. Robot control systems are responsible for guidance and navigation and control of robots in changing dynamic environments. Position and force/torque control is still an important problem despite major advances made in that field. Nonlinear control systems could be identified as one of the key problems in that area. The second is control of autonomous systems.

Perception systems are based on sensors that supply information about the robot's surroundings. Clearly, an understanding of the operating environment is essential for an intelligent, autonomous robot. Information from sensors are integrated (Sasiadek, 2002) in the process called sensor/data fusion. This sensor fusion process became one of the most important problems related to intelligent robotics (Sasiadek and Wang, 2003).

The key problem in robot's mechanical design is human-robot interface and interaction. Humans are used to interact with other humans, animals or machines that are designed and made to human "scale". Very often this human scale is seen in the perspective of customs and evolutionary changes and adaptation. Contemporary robots vary because their design and purpose. In general, the robots could be divided into two groups: stationary and mobile. The stationary robots are designed in form of manipulators. These manipulators have different types of kinematics but the most often they use the "arm" kinematics that is similar to the biomechanical design of our own hand. However, very often mobile robots do not have any "friendly" interface and it is difficult for humans to interact and communicate with them. The major challenge for robots designers is to make them easy to use by, and communicate with humans.

Also, the application of new micro-electromechanical systems (MEMS) to robots poses challenging design problem. MEMS are systems (components) that integrate sensors and actuators on the silicon substrate and are made using the microelectronics and micromachining technology. The key problems are the large scale manufacturing, reliability, and successful integration of MEMS with microprocessors.

\subsection{Human-Machine systems}

Over the last years a fast and steady increase in complexity of modern industrial systems can be recognized. This complexity is characterized by a huge increase of functions and data, by linking single machines to system networks thus extending the mental workspace and by more and more replacing visible mechanical by invisible computerized functions. In many research projects it can be proven that these developments have become key problems for human operators, and the integrity of the human decisions and control have thereby suffered. Whereas a lot of research work was carried out over the last decades in high-risk areas like nuclear power generation or aviation, the problems were recognized much later in the more low-risk areas.

Today's situation is characterized by replacing unique control hardware by standardized hardware platforms mostly based on PC technology at least at the user-interface level. Industrial surveys show that PC-based interactions using Windows-like operating systems, industrial pointing devices and touch screens are becoming more and more an industry standard. But the functions offered by this sophisticated base equipment lead developers to implement hundreds of functions instead of usercentered solutions. Unfortunately, most of these developers are not educated to implement the basics of ergonomic design and the shorter development cycles increase the pressure on them. On the other hand many manufacturers still believe that more functions and nicely colored screens lead to better sales.

But the situation is now rapidly changing. The manufacturers of complex technical equipment are recognizing more and more that "functionalities" is a very short-sighted marketing strategy. And industrial customers recognize that a human-centered HMS reduces training effort and error rate, leading to a more efficient use of the expensive equipment. Most of the global technology leaders have installed usability labs including the necessary trained staff over the last 5 years and this trend is growing.

\subsection{Cost Oriented Automation}

Controlling Distributed Collaborative Engineering, i.e. to control common work over remote sites, is now an emerging topic in Cost Oriented Automation. Therefore cost effective product and process realization has to consider several aspects regarding automatic control: Virtual Manufacturing supporting Integrated Product and Process-Development, Teleor Web-based Maintenance (Cost Reduction with e maintenance Systems in Manufacturing), and SMEoriented agile manufacturing. Agile manufacturing here is understood as the synthesis of a number of independent small or medium sized enterprises (SME's) forming a network to join their core skills, competencies and capacities to be capable of operating cost effectively in a competitive environment of continual, and unpredictable, changing customer demands.

As mentioned in the introduction life-cycle management of automation systems is important regarding the cost of ownership. The complete production process should be considered respecting its performance where maintenance is the most 
important part regarding the cost. Maintenance is still today either reactive or blindly proactive. Degradation of parts is largely invisible to human users, even though a great deal of technology has been developed that could make such information visible. Many sophisticated sensors and computerized components are capable of delivering data about the machine's status and performance. When machines are networked and remotely monitored, and when their data is modelled and continually analyzed with embedded systems, it is possible to go beyond mere "predictive maintenance" to intelligent "prognostics" (condition based maintenance). Morel (2004) considers the performance of the complete automation system, that interests the owner respecting the cost, rather than the control performance only; i.e. a compromise between cost of maintenance and cost of standstill of the automation system has to be found. The advantage of e-maintenance was discussed by Lee (2004), and here human-human collaboration has to be made effective.
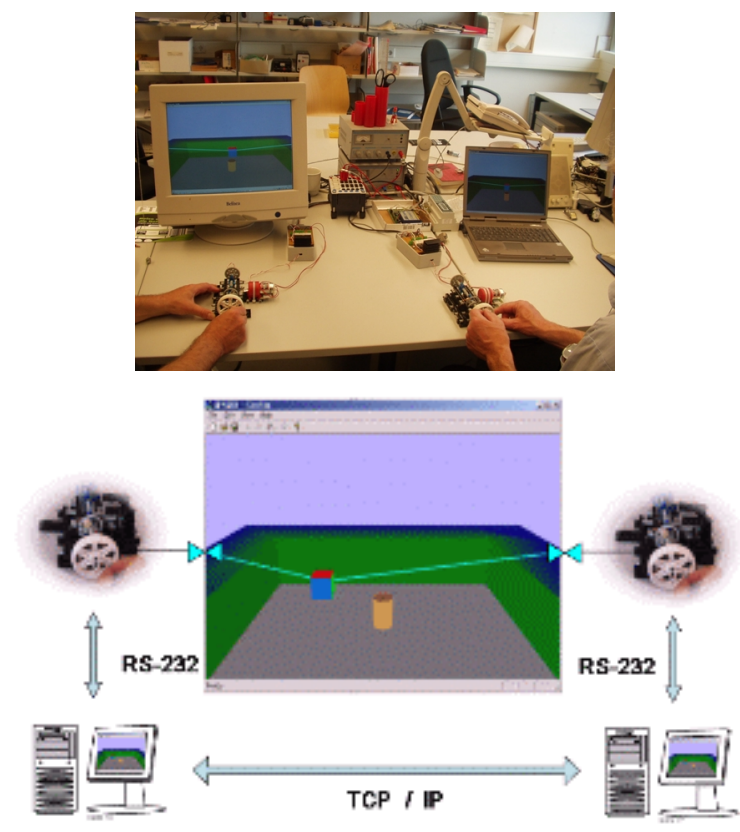

Fig. 1. Moving a virtual mass with two momentum handles (Bruns \& Yoo, 2004).

Human-Human Collaboration among others has been identified as an emerging area for Automatic Control. Supporting cost effective Human-Human Collaboration in networked enterprises is a challenge for cost oriented automation. With the trend to extend the design and processing of products over different and remotely located factories, the problem arises how to secure an effective collaboration of the involved workforce. The usual face-to-face work will be replaced at least partly if not totally by computer mediated collaboration. The development and implementation of information and communication technology, suitably adapted to the needs of the workforce and facilitating remotely distributed collaborative work is a challenge to engineers.
Information mediated only via vision and sound is insufficient for collaboration. In designing and manufacturing it is often necessary to have the parts in your hands. To grasp a part at a remote site requires force (haptic)-feedback in addition to vision and sound. Consider for example remote service for maintenance. Low cost force feedback devices are under development, and these can be used in so called mixed reality: Bruns \& Yoo (2004) demonstrated (Fig. 1) the movement of a virtual mass on a table at a computer-screen with force feedback handles via the internet using hyperbonds, a sensinggenerating interface (Bruns, 2003).

\section{ACCOMPLISHMENTS AND TRENDS}

\subsection{Components and Instruments}

In the area of factory automation (see proceedings of the IEEE International Conference on Emerging Technologies and Factory Automation) we may expect a future intensive use of intelligent components in applications ensuring the monitoring of the environment (see Akyildiz et all, 2002). The research in new solid state sensors using microelectronic technologies, embedding signal processing and pattern recognition functionalities will probably evolve soon in the production of electronic noses able of detecting and identifying numerous varieties of harmful gas like the $\mathrm{CO} 2$ or the cooling gases (see Nagle et al, 1998). These sensors will become essential parts of a monitoring system aiming to fight against atmospheric pollution. It also indicates an open research topic for additional applications in areas like medicine, leading to a massive production of biomedical wearable devices (see proceedings of the IEEE International symposium on BIO-Informatics and Biomedical Engineering and. Schwiebert et al, 2001).

In the biomedical field, the current applications are mainly focused on the remote monitoring of physiological signals like blood pressure, electrocardiogram, etc. But intelligent components can also contribute to the monitoring and control of the muscular movements or the artificial heart, to the detection of brain malfunction and so on.

As far as Components and Instruments for transportation/automotive are concerned, one of the most recent and challenging topics for research activities is certainly the very important advances related to Driver Assistance Systems (ADAS). These include several functions related to the vehicle longitudinal and lateral control: Lane Departure Warning (LDW), ACC (Adaptive Cruise Control), Lane Keeping, Stop and Go Function etc. Development of these new functions is strongly related to sensor technology push, the tremendous increase of real time processing capabilities, and of course the latest advances in terms of signal processing, image processing and so on (see 
Walldorf and Gessner, 2003 and Krueger and Gessner, 2002). The first examples of these new systems are now coming to the market, for example Daimler Chrysler is commercializing a Lane Departure Warning systems for their trucks (ACTROS).

Some of the main research and developments trends for the very next years are:

- To provide new generation of sensors (video sensor-cameras, RADAR, LIDAR, etc) able to fulfill automotive specific requirements: low cost (some Euro!), high quality, severe environmental constraints (for example temperature ranges from $40^{\circ}$ to $105^{\circ}$, low sensitivity to EMC, ...), capacity to operate in adverse situations (for example, for camera, ability to operate by night, by day, with very contrasted scenes, ...).

- To provide sophisticated data fusion, software processing, and algorithms able to extract from complex information more synthetic data (for example driver eye gaze or eyelid movement, driver posture and activity, obstacle detection and classification, etc).

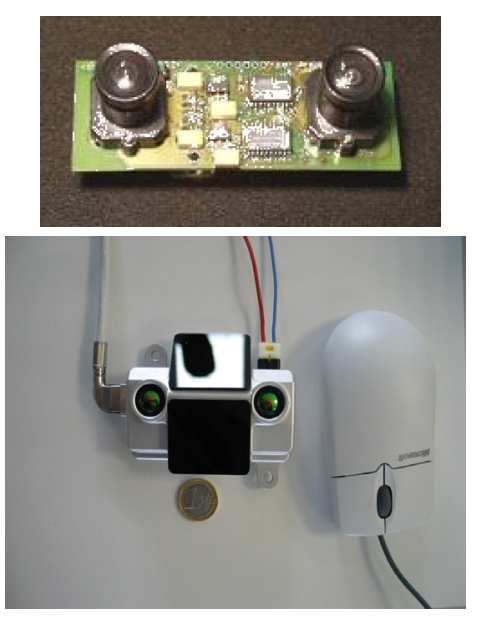

Fig. 2: Example of Integrated low cost stereovision camera module.

\subsection{Mechatronics}

Many industries over the last decade have made great strides towards mechatronic solutions, some of which link strongly to developments identified elsewhere in this article, especially those related to Components and Instruments. Key industries where mechatronic products are fundamental to their current production technology are:

- Automotive: active steering for automobiles is now in series production with BMW; the electrohydraulic brake (Bosch, Mercedes Benz).

- Combustion engines: the common rail injection system for Diesel engines and also the injection systems for gasoline engines.
- Data storage: sophisticated but extremely costeffective magnetic and optical storage systems based upon sophisticated embedded control highly integrated with the mechanics of the products.

- Tilting technology in trains enabling higher speeds through curves; active secondary suspensions to give improved ride quality.

- And many others ...

Progress is being made towards tackling a number of the key skills issues in mechatronics. Universities have recognised industry's needs for multidisciplinary engineers and are increasingly offering targeted mechatronic undergraduate programmes that satisfy some of the multidisciplinary skills requirements, and through this and other trends there is undoubtedly a greater awareness within industry of the importance of the mechatronic approach than there was (say) 10 years ago.

Design tools are progressively improving in their ability to handle the multi-domain modelling in a usable manner, although truly multi-objective optimisation capabilities remain limited (and where they are provided they are often under-exploited). In general modern design tools are better at providing the full-complexity simulation models than they are the appropriate simplified models needed for control design; even though formal model reduction techniques are available, they need considerable translation for use in a practical design context, in addition to which they are primarily based upon linear(ised) models because non-linear model reduction is still an open research problem.

The overall research trends must therefore be towards more systems-oriented design methodologies and software tools that can provide real support for the burgeoning range of mechatronic products and processes, in particular bringing in some of the increasingly critical requirements related to fault tolerance, human factors, etc.

\subsection{Robotics}

There are number of major achievements and trends in robotics. One of them is development of autonomous systems. In particular, the rapid progress in development of autonomous Unmanned Aerial Vehicles (UAV) (Jardin and Bryson, 2001; Ollero and Merino, 2004; Wu et al, 2004) with sophisticated formation flight control (Binetti et al, 2003; Patcher et al, 2001) is a significant accomplishment.

Several unmanned aerial vehicles were tested in USA and Europe. Some of them are small and the other large vehicles. They move slow (helicopter like UAV) or fast (hypersonic UAV) and they represent one of the fastest growing area of advanced 
technologies at present time. Fig. 3 shows two robotic aircrafts (http://www.uavforum.com/).

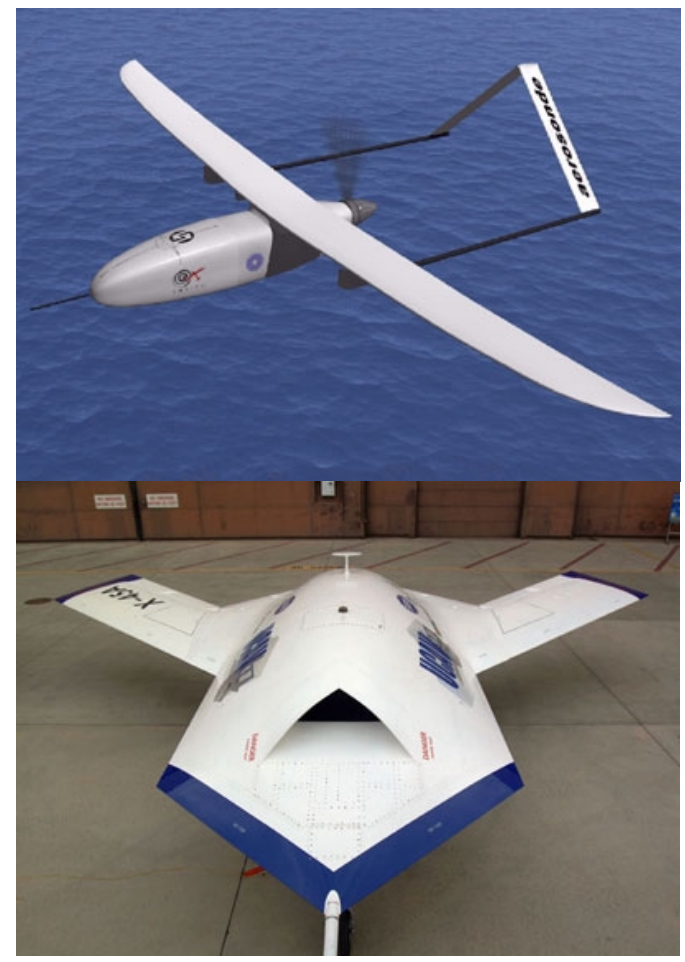

Fig. 3: 3a Aerosonde Robotic Aircraft, 3b Boeing X45 UCAV.

Yet another important and promising sub-field of robotics is underwater robotics. The underwater robots are used in underwater exploration, mining, oil industry and military application, e.g. mine detection. Many Unmanned Underwater vehicles have been also developed (see for example Figure 4).

The second major achievement is in telerobotics, especially in medical applications. Medical surgery performed from a remote location has now become a reality. For example the first successful surgery across the continents took place in 2001 when an American doctor from New York removed the diseased gallbladder in a 68 year old patient in Strasbourg, France.

The third major progress and accomplishment can be identified is space robotics. The exploration of outer planets (e.g. Mars) using mobile robots is a spectacular success (http://www.jpl.nasa.gov/videos). The Mars exploration mission is still underway (Fall 2004) using mobile robot explorer named Spirit. The Space Station built at the Earth orbit is being equipped with the Canadarm2, seven degree of freedom robotic manipulator that will help to further develop and maintain the space station. The Mars exploration mission is still underway (Fall 2004) using mobile robot explorer named Spirit.

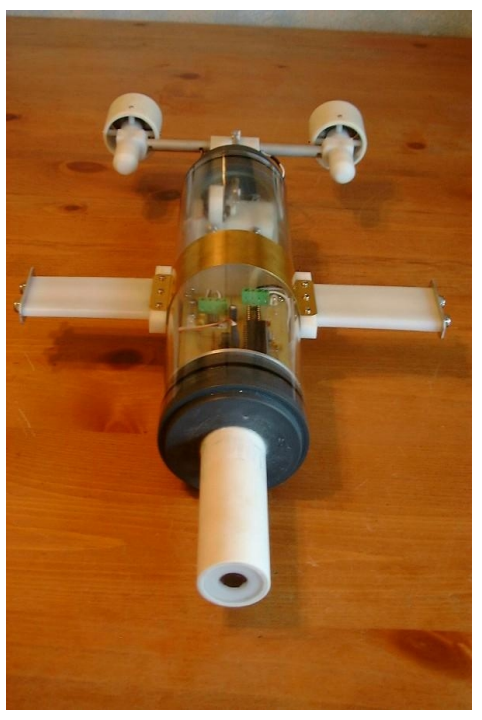

Fig. 4: HUV Underwater Robot.

The Space Station built at the Earth orbit is being equipped with the Canadarm2, seven degree of freedom robotic manipulator that will help to further develop and maintain the space station. The Canadarm2 is fitted with yet another two-arm robotic manipulator called Special Purpose Dextrous Manipulator (SPDM) shown in Fig. 5. The Personal Space Assistant (PSA) a free flying robot is being developed by the NASA Ames Centre.

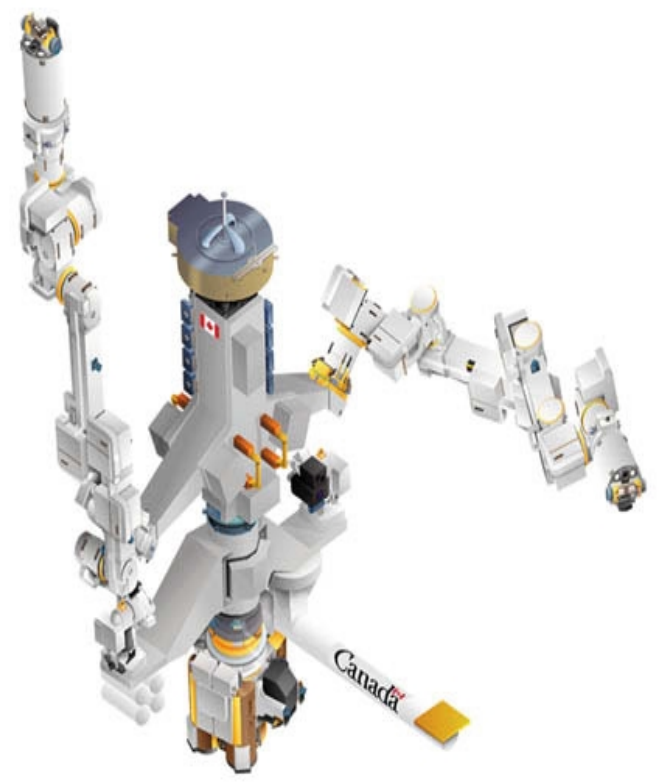

Fig. 5. Special Purpose Dextrous Manipulator (SPDM)(http://www.mdrobotics.ca/wwdframe.ht $\mathrm{ml})$.

The development of humanoids is also an emerging trend in robotics (http://www.humanoidrobots.org).

Furthermore, service robotics is an emerging industrial application. Many such robots have been described in (http://www.service-robots.org). 
Finally, it should be also noted the developments of multi-robot systems (Arai et al, 2002). These developments include cooperative manipulation, and the coordination of multiple autonomous vehicles. Different systems and technologies for the coordination of fleets of ground and aerial vehicles have been developed. The cooperation of multiple autonomous robots is an active research topic in the last ten years (Cao et al, 1997) with many potential applications. For example, in Ollero et al (2005) a system for the coordination of multiple heterogeneous aerial vehicles with application to forest fires is presented.

\subsection{Human-machine systems}

Driven by the change of attitude of manufacturers recognizing that "functionalities" is a very shortsighted marketing strategy, and of industrial customers recognizing that a human-centered HMS leads to more efficient use of the expensive equipments as described in section 2.4 of this report, an increasing interest in development and implementation of human-centered user interfaces has been noticed within industry. Starting with consumer areas like mobile phones or smart homes and moving into the broad area of web-site usability, the ideas of user-centered HMS are nowadays also accepted by other parts of industry like capital goods and the automotive industry.

Over the last years a lot of research work was carried out in the field of human-centered system design. But compared to other scientific fields, the area is very broad and influenced by several sciences:

- The social demands are mainly pushed by socialscientists under the subject of socio-technical system design.

- Researchers with a psychology background focus their work under the subject of cognitive engineering.

- The work ergonomics tend to call their subject usability engineering.

- The computer science community talks about system engineering and intelligent user interfaces based on principles from the AI (Artificial Intelligence) field.

But, as a German survey among several hundred equipment manufacturers in 2003 showed, $75 \%$ of the industrial user interfaces are designed by engineers who are facing many theories which go far beyond their actual need. The survey also revealed that industry is still thinking and structured in simple traditional categories like hardware and software engineering. Therefore, in 1997 the major German societies for computer sciences (GI), ergonomics (GfA), information technology (VDE-ITG) and automation (VDI-GMA) established the new subject Useware Engineering (UE).

The term useware was carefully chosen in analogy to hard- and software and describes all hardware and software components to implement as well as methods to design human-machine-systems (Oberquelle, 2002; http://www.useware-forum.de). It was felt that one catch-word will help to better identify the community dealing with all aspects of HMS. After six years, the latest survey showed that this subject is well accepted in the German industry and leads to the necessary discernment that useware is at least of equal importance as hard- or software.

The described situation should not be misinterpreted as a competitive situation; it rather demonstrates the interdisciplinary orientation of this field. All approaches have the same goal: to design complex technical systems based on capabilities and needs of human users.

Looking into the more technical fields, a rapid move towards a broad use of modern PC-based equipment is to be recognized. The surveys show that the industry is using more and more the well-proven and standardized hard- and software components from the PC-market. There are VGA/XGA-color displays with or without touch input, mouse-devices, windows-like operating systems, browsers etc. These technologies allow the implementation of very powerful and also cost-efficient HMS.

Whereas the majority of industrial users are still on the PC level, the leading players are moving towards new technologies. One very recent trend is the increasing use of mobile computing devices in the industrial areas which nowadays offer a powerperformance profile that is sufficient even in high reliability application areas. Whether these devices are smart-phones, PDAs or dedicated mobile computers, they will be used as a replacement for or additional front-end to traditional operating panels. Communication between the controllers and these devices will use the wireless networking standards available today like WLAN, Bluetooth or UMTS. The increased mobility of the worker will surely lead to new forms of work in a sense that the interaction location is no longer identical to the process location. This will require new work and data security regulations. One problem is user authentification. Besides the well-known knowledge-based methods (e.g. passwords) new biometrical methods (e.g. iris scan, finger print scan) are close to industrial use. Mobile devices will not only be handheld but also become an integral part of the human (work) clothes. These real wearable systems will bring new forms of interaction to the user.

When using mobile devices the location of the user in relation to the process equipment becomes very important. Therefore, these devices must correspond to location based services offered by the environment. While the GPS system is limited to 
outdoor applications with a resolution between 5 and 30 meters, new systems for indoor use with resolutions down to centimeters or even millimeters are required. And for augmented reality applications they must offer precise angle information besides the lateral position data. Here, 4-5 different location detection technologies based on RF, US and IR methods have been developed today, but they are still in a laboratory phase.

In many situations the user needs support and supervision to guide him through a specific situation or prevent him from irreversible actions. Here, intelligent agents seem to be the appropriate solution. Linked together to multi-agent systems, they can watch and model the individual user, take over many routine tasks and give advice in unclear situations. A prerequisite for doing this is a task and user model. Whereas only a small part of such a model is static and predefinable, the major part can only be built online by watching the user and his typical tasks. Here the AI field of knowledge engineering plays the key role.

Mobile devices and embedded systems, wireless adhoc networks and location based services are basic building blocks for the next generation of user interfaces. This will be the pervasive computing, ubiquitous computing or ambient intelligence era. The future systems will be invisibly integrated into everyday devices. The users will interact with those devices human-like via natural speech, gesture or mimics (Johannsen, 2002).

Evaluating the research and application of HMS worldwide, it must be stated that until today nearly all activities are to be found in the industrialized countries. Here, the existing technology fulfils the basic needs of the market. And with nearly all producers fulfilling these needs other differentiating topics become important for successfully selling equipment. Besides the joy-of-use factor as one important topic, more and more the ease-of-use factor is recognized as being a topic of equal importance.

Another disappearing phenomenon is the northsouth-gradient. Whereas ergonomics and HMS have a long tradition in the more northern countries, in the southern countries the interest is still developing. But the globalization of markets and the establishment of technical standards, e.g. set by the EC or by ISO, will force all nations to accept usability issues.

\subsection{Cost Oriented Automation}

Integrated Product and Process Development as a cost saving strategy has been partly introduced in industry. However, as Nnaji (2004) mentioned, lack of information from suppliers and working partners, incompleteness and inconsistency of product information/knowledge within the collaborating group, incapability of processing information/data from other parties due to the problem of interoperability hampers the effective use. Hence, collaborative design tools are needed to improve collaboration among distributed design groups, enhance knowledge sharing, and assist in better decision making.

Condition based maintenance strategies has been also partly implemented. The trend is to merge on-site and remote intensive infotronics technologies (Lee, 2004) in order to recover expected performances as well as to face increasing demands on performance (agility, interoperability, reactivity, configurability, security certification, failure recovery).

Both strategies will be integrated to achieve a reduction of life-cycle costs, and enhancing the overall performance (Morel, 2004).

Energy Saving and therefore cost saving with Plantand Building-Automation is a relevant topic for cost oriented automation. The trend to decentralize energy support aims for intelligent distributed control.

Automation in Mining, Agriculture, and Transport with field-robots is a developing area for cost oriented automation. Suitable automation saves energy, improves the security of the workforce, and is more efficient than manual work, therefore enhancing the competitiveness of factories. The problems to solve here are low cost tele-operation or tele-manipulation with force feedback, and the maintenance. Scheduled maintenance has to be replaced by predictive maintenance based on condition monitoring. Sensors with local information processing to minimize the flood of data to be sent to the supervision computer, as well as data fusion processes are saving cost.

The development of collaborating systems is the trend for the future for automatic control in general, and particularly when considering their cost effectiveness. Collaboration of human operators and automation systems like robots are under development for achieving more flexibility in production and for saving cost when avoiding repeated reconfiguration of the systems. The development of collaborative robots (Cobots) or Intelligent Assisting Devices (IAD) was motivated by ergonomic problems in assembly of parts, where its weight endangered long-term the human body (Surdilovic et al, 2003).

Smart Devices (sensors, actuators) with local information processing in connection with data fusion is in steady development (Zayed et al, 2004) regarding cost reduction of components as well as with application in several fields (automotive, robotics, mechatronics, manufacturing). 


\section{FORECAST}

\subsection{Components and Instruments}

The design of sensors, especially biomedical sensors, introduces new challenging constraints. The design of a biomedical sensor is for example strongly affected by the restricted shape, size and the weight the sensor must have. Power restrictions apply themselves, due to the inability to supply the energy through the traditional wires. Implanted biomedical sensors are also expected to be operational for an extended period of time.

The capability of exchanging the information through a wireless communication is essential. In fact, the application of wireless sensor networks is an important trend with application to many different fields. The Confidentiality of the transmitted information is finally required to ensure the integrity of this information against potential doubtful handling. To solve these problems, various methods can be envisioned, including fault tolerance, data encryption, data compression, dynamic power management and so on. The use of low-power microprocessors can also be a solution to the power limitation. The high computational power demand that could result when implementing these treatments can also induce the development of custom hardware architecture. In that case, the co-simulation technique of hardware/software solutions is probably the essential technology that will ensure a development process mixing the functional aspects with the performances aspects to determine the suitable architecture for the sensor.

In the transportation/automotive application field the most advanced research and development activities for the future will be obviously to better understand interactions between driver, vehicle (including assistance and information functions) and the environment. In Europe several EU funded projects including industrial and public research groups are strongly involved on that topic (SENSATION: European project 6th PCRD IST program $n^{\circ} 507231$; AIDE: European Project 6th PCRD, IST Program $n^{\circ}$ 50767; PREVENT: European Program 6th PCRD, IST Program n507075).

\subsection{Mechatronics Systems}

Despite being a relatively new area in terms of real industrial involvement, there has been an enormous increase in the number of products and processes which can now be considered to be mechatronic. The increasing awareness of future products and processes that can be facilitated by the application of mechatronics will continue to grow, and many industrial companies, research organizations and university departments have reorganized to ensure that the maximum exploitation possibilities are realised.
In particular the industrial areas mentioned in Sect 3.2 will continue to be developed to provide higher mechatronic implementations: automobiles will progressively take up concepts such as steer-by-wire, currently only at the experimental stage; data storage systems will adapt towards new concepts that still require sophisticated control and data processing; the use of mechatronics in trains will progress towards active control of running stability and, possibly, new mechatronic vehicle concepts; and so on.

Future systems will see further synergies between mechanics, sensing and actuation, greatly enhanced by embedded electronic "system-on-chip" technologies which will continue to shift the costeffectiveness balance towards solutions that incorporate advanced electronic processing. A key complementary technology for mechatronic products is MEMS, linked of course to developments in Components and Instruments.

A future possibility is mechatronics of a more hierarchical nature, e.g. high functionality mechatronic systems constructed from a set of mechatronic sub-systems providing distributed processing functions combined with central coordination. These would include smart materials to make up the mechanical structure, sophisticated MEMS sensors, self-diagnosing actuators and complex, centralized information processing capable of adaptation and reconfiguration to maximize performance and reliability at minimum cost.

Mechatronics is already an enabling system-level technology that cuts across many industrial sectors, and as the major challenges are tackled and overcome it will become more and more a normal part of the engineering design process, so much so that purely mechanically-based solutions will become the exception. The implications of this trend raise questions about the future role of mechatronics: it is (arguably) a paradigm rather than a science, and as it increasingly becomes accepted as the normal approach to engineering the role of mechatronics training and technical support activities, and indeed of the word itself, need to be developed accordingly.

\subsection{Robotics}

Robotics is a relatively new field of engineering but seems to enjoy lasting interests of researchers and scientists as well as the public. Initially, i.e. in the 1970s and 1980s, development of robotics was driven mostly by manufacturing applications, and therefore there was a broad interest in manipulator type robots. In the 1990s and in the first decade of this century the development of robotics has been driven more by the needs in aerospace, service and biomedical industry. 
The fast pace of progress in robotics seems to have been sustained for a long time and in the future, the pace of development may even be accelerated. It is expected that autonomous robots such as UAVs, UGVs, UXV (their combination), service robots, e.g. "personal robots", mining vehicles and other, will be brought to a very high level of sophistication. At the same time, some of the robotics methodologies such as perception and sensor fusion will be broadly used, not only in robotics but also in all so-called "intelligent" machines.

Another field of robotics destined for rapid progress and development is biomedical robotics.

The development of micro and nano robots is also a hot research topic involving challenges in different fields. These robots have many potential future applications, and could have a significant impact on the future developments in robotics.

\subsection{Human-machine systems}

As already mentioned, technological advancements in the field of electronics, communication and information technology have led to new interaction methods. This can be observed not only in office applications, but also in industrial areas. An on-line Delphi survey conducted by the IFAC Technical Committee on Human Machine Systems in 2002 on a worldwide basis gave a vivid picture of the importance and development of new technologies for Human-Machine Interaction (HMI) over the next 30 years(http://www.unikl.de/pak/ifac/ifacdelphiresults. html). The main topics of the survey were technologies for interaction, visualization and user authentification, intelligent user interfaces, emotional/affective engineering as well as trends in work organization.

The survey revealed that among interaction technologies such as tactile, gesture, gaze, speech, mimic, mind control and multimodal interaction, speech interaction will be the most important technology in the future. This is probably because of the ease and flexibility it offers that resembles natural human-human communication. The survey predicts that full development of speech interaction will soon be reached (2005). Another technology that will play an important role in the future of HMI is multimodal interaction, i.e. a combination of more than one interaction modals (see Figure 6). This might lead to machines having artificial eyes, ears, mouth, skin etc.

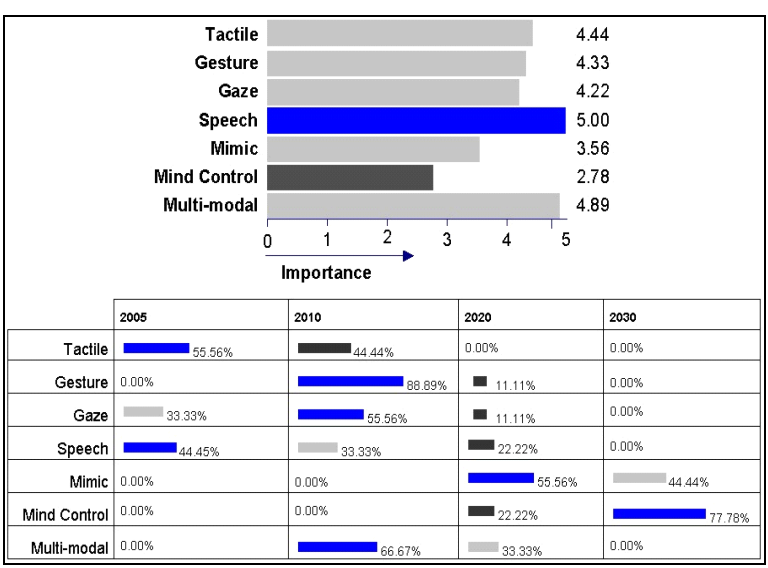

Fig. 6: Results of the survey on the importance and development of interaction technologies

The most important visualization technology in HMI in the future will be augmented reality. This technology is based on $3 \mathrm{D}$ visualization, which was considered to be almost equally important for HMI in the future. According to the survey, these technologies should be fully implemented by the year 2010. According to the survey other visualization technologies such as virtual reality, head-mounted display and free form display were not thought to be of much importance for HMI in the future (see Figure 7).

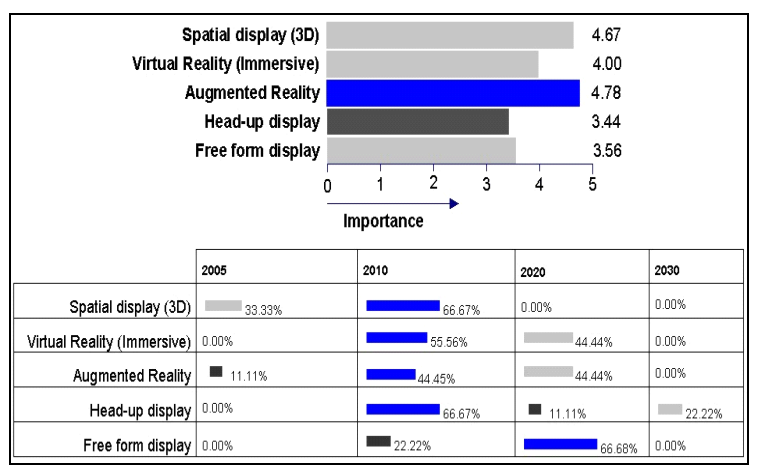

Fig. 7: Results of the survey on the importance and development of visualization technologies

As machines become complex with sophisticated functionalities, secure user authentification is needed. Though normal user authentification methods like password and chip card will still be in use, biometrical methods like iris scan, fingerprints, and voice identification are getting more important and might be fully implemented by 2015. They will replace or supplement the now common methods mentioned above.

Which intelligent User Interface (UI) will be important for HMI in future? Will it be intelligent agents, avatars, emotional (affective) UIs or personalized UIs? Results showed that intelligent agents and personalized UIs could be important in the future of HMI. While intelligent agents could be fully implemented by year 2005 already, it might 
take 5 more years for personalized UIs to reach their state of the art.

And which role will emotional/affective engineering play in future HMI? Will recognizing and expressing emotions be important for future technical systems? The survey showed that technologies for recognizing emotions will be more important than those for expressing them. Research in these fields should be closed by year 2010, so the survey says. A sub-topic to the field of emotions is surely the use of music or - in a broader sense - sounds to carry information.

Technological advancements have not only changed machines, but also the organization of work. New means of group interaction such as shared workspaces and the virtual control room have emerged. When considering the future of HMI, shared workspaces will be most important for group interaction and will soon be standard.

Concluding the results from this survey, the future of HMS will move into the direction of human-human like interaction. That means the interaction will be more informal and error-redundant due to parallel processing of several modalities with redundant information. It will also become more pleasant (Joy of use) to the user due to the emotional capabilities of the technical devices. And the interface will disappear from the technical outer shell into the inner realization of technical devices.

\subsection{Cost Oriented Automation}

The recent development of Ubiquitous Computing deserves attention in the Cost Oriented Automation field. It involves the close coupling of physical objects with information processing.

Ubiquitous automation might be an important field of future work regarding cost savings, involving: Advanced sensor-actuator interfaces (so called Hyper-Bonds), Unified models of mapping physics to information and vice versa (bond-graphs, and Petri-Nets), mixed reality frameworks of distributed model-view-control, mixed reality multi user environments (human-human collaboration), real time simulation, and more consideration of the importance of enjoyment, bridging perspectives of toys and tools.

\section{CONCLUSIONS}

This paper has outlined the current key problems, accomplishments and forecasts in control technologies including components and instruments, mechatronics, robotics, human-machine systems and cost oriented automation.

Two different general trends can be identified. The first one is development of embedded components and systems integrating perception, control and actuation functions that can be used, eventually, in a transparent way. This trend has been motivated by recent technological developments (hardware integration, MEMS, ...) and the requirements imposed by new applications (vehicles, autonomous systems, consumer products, biomedical systems, engines, manufacturing ...). MEMS and nanotechnologies could be one of the most significant control demanding fields in the future.

The second is the distribution of sensing and control functions. Thus, distributed control systems with wire and wireless connections between components and embedded controllers have emerged in the last years, also pushed by the developments in communications technology and new applications (Tele- applications, distributed manufacturing, protection of people and environment, home automation, ...).

Thus, one of the main general forecasts/trends is the integration of control and perception components into embedded systems that can be networked using wire or wireless technologies, leading to "cooperating objects", with sensing and/or actuation capabilities based on sensor fusion methods that allow full interaction with the environment.

The main trends in the applications of these technologies are medicine (surgical devices, medical instruments), new cars, vehicles and transportation technologies, autonomous systems technology (UAV, UGV, AUVs, multi-robot systems), industrial robotics and automation technology, technologies for safety critical and hostile environments (space, disaster remediation, defence, ...), security technologies, energy savings (mixed energy sources, small scale energy systems) and biotechnologies.

The consequences of communications can be considered two-fold: firstly, communication technologies demand the application of new control systems (i.e. network control, wireless devices control). Secondly, communication is a key technology for the implementation of control systems in the above mentioned applications.

The general requirements in most of the above mentioned technologies are: performance, reliability, cost, ease of use and maintainability, and these must remain the principal focus of future research in the all the subjects relevant to the area of Mechatronics, Robotics and Components.

\section{REFERENCES}

Akyildiz I.F., W. Su, Y. Sankarasubramaniam and E.Cayiri, (2002). A Survey on Sensor Networks. IEEE Communication Magazine pp102-114 August 2002.

Arai T., E. Pagello and L.E. Parker (2002). Advances in Multi-robot Systems. IEEE Transactions on Robotics and Automation. Vol. 18, No. 5. 
Binetti et al. (2003). Formation Flight Optimization Using Extremum Seeking Feedback, AIAA Journal of Guidance, Control and Dynamics, Vol. 26, No. 1, pg 132-142

Bruns, F. W. (2003). Hyper-Bonds. Human Skills Oriented Systems Design with Mixed Reality. In: Proc. Proc. of 6th IFAC Symposium on Automated Systems based on Human Skill and Knowledge. Elsevier Ltd., Oxford.

Bruns, F.W., Y. H. Yoo (2004). Realtime collaborative mixed reality environment with force feedback. In: Proc. of the 7th IFAC Symp. On Cost Oriented Automation, Ottawa, Canada. Elsevier Ltd., Oxford. In press.

Cao Y.U., A.S. Fukunaga and A.B. Kahng (1997). "Cooperative mobile robotics: antecedents and directions". Autonomous Robots, 4, 1-23.

FET (2003) 5th International Conference on Field Buses and their Application IFAC. July 7-8, 2003, Aveiro, Portugal.

Grace R.H., (2002). Markets Opportunities for MEMS/MST in Automotive applications. In Advanced Microsystems for Automotive Yearbook 2003; pp 3-12; Springer-Verlag, March 2002 Berlin (Germany).

http://www.humanoidrobots.org

http://www.huv.com/uSeeker/index.html

http://www.jpl.nasa.gov/videosp://www.mdrobotics. $\mathrm{ca} /$ wwdframe.html

http://www.service-robots.org/CleaningRobots.php

http://www.uavforum.com/

http://www.useware-forum.de

IEEE International Conference on Emerging Technologies and Factory Automation (ETFA). Lisboa, Portugal, September 2003.

IEEE International Symposium on BIO-Informatics and Biomedical Engineering (BIBE). Arlington Virginia, November 2000.

IFAC TC 4.5 Delphi survey (2002). http://www.unikl.de/pak/ifac/ifacdelphiresults.html

Jardin and Bryson (2001), "Neighboring Optimal Aircraft Guidance in Winds", AIAA Journal of Guidance, Control and Dynamics, Vol. 24, No. 4, pg 710-715

Johannsen, G. Ed., (2002). Proc. of the 8th IFAC Symposium on Analysis, Design and Evaluation of Human-Machine-Systems, Kassel 2002. Elsevier Publishing.

Krueger S. and W. Gessner, (2002). Advanced Microsystems for Automotive Yearbook. Springer-Verlag, March 2002 Berlin (Germany).

Lee, J. et al (2004). Infotronics Technologies and predictive tools for Next-generation Maintenance systems. In: Proceedings of the 11th IFAC Symposium Information Control Problems in Manufacturing - INCOM 2004. Elsevier Ltd., Oxford.

Martensson L. and J. Cernetic (2002). IFAC 2002 Milestone Report on Social Impact of Automation. Proc. of the 15th IFAC World Congress, Barcelona.

Morel, G. et al (2004). Performance Oriented System Engineering: Rationales, Experiments and open
Issues. In: Preprints of 7th IFAC Symposium on Cost Oriented Automation, Ottawa, Canada.

Morpha (2003). Kommunikation, Interaktion und Kooperation zwischen Menschen und intelligenten anthropomorphen Assistenzsystemen. http://www.morpha.de

Nagle T., R. Gutierez-Osuna and S.S. Schiffman (1998). The how and why of Electronic Noises, IEEE Spectrum, 35, 9, pp 22-34.

Nnaji, B. et al (2004). Cost effective Product Realization. In: Proceedings of 7th IFAC Symposium on Cost Oriented Automation, Ottawa, Canada. Elsevier Ltd., Oxford. In press.

Oberquelle, H., (2002). Useware Design and Evolution: Bridging social Thinking and Software Construction. In: Dittrich, Y., Floyd, C., Klischewski, R. (Eds.): Social Thinking Software Practice. MIT-Press, Cambridge, London.

Ollero A., G. Morel, P. Bernus, S.Y. Nof, J. Sasiadek, S. Boverie, H. Erbe, R. Goodall (2002). "Milestone report of the manufacturing and instrumentation coordinating committee: from MEMS to enterprise systems". Annual Reviews in Control, Elsevier Science, Vol. 26, Part 2, pp 151-162, 2002

Ollero, A. and L. Merino (2004), Control and Perception Techniques for Aerial Robotics, Annual Reviews in Control, Vol. 28, pp 167-178.

Ollero, A. et al. (2005). Architecture and perception issues in the COMETS multi-UAV project. IEEE Robotics and Automation Magazine. To appear.

Pachter et al (2001), "Tight Formation Flight Control", AIAA Journal of Guidance, Control and Dynamics, Vol. 24, No.2, pg 246-254.

Sasiadek, J.Z. (2002) "Sensor Fusion", Annual Reviews in Control, IFAC Journal, No. 26, 2002 pp 203-228.

Sasiadek, J.Z. Wang, Q. (2003), "Low Cost Automation Using INS/GPS Data Fusion for Accurate Positioning”, Robotica, Vol.21, No.12, May-June 2003.

Schwiebert L., S.K.S.Gupta, and J.Weinmann (2001). Research challenges in Wireless Networks of Biomedical sensors. ACM SIGMOBILE, Roma, Italy, July 2001.

Surdilovic, D., R. Bernhardt, L. Zhang (2003). New

intelligent power-assist systems based on differential transmission. Robotica, Vol. 21, pp. 295-302.

Walldorf J. and W. Gessner editors (2003). Advanced Microsystems for Automotive Yearbook. Springer-Verlag, May 2003 Berlin (Germany).

Wu, H. et al (2004). "Micro Air Vehicle: Configuration, Analysis, Fabrication and Tests", IEEE Transaction on Mechatronics, Vol. 9, No.1, March.

Zayed, M., Boonaert,J., Bayart, M. (2004). A low cost stereovision sensor for non-contact measurement purpose. In: Proceedings of 7th IFAC Symposium on Cost Oriented Automation, Ottawa, Canada. Elsevier Ltd., Oxford. In press 